\title{
Performance of Trellis-Coded Direct-Sequence Spread Spectrum with Noncoherent Reception in a Fading Environment
}

\author{
Victor Wen-Kai Cheng and Wayne E. Stark
}

\begin{abstract}
In this paper, we consider the performance of different coding schemes for direct-sequence spread spectrum (DS-SS) in a nonselective Rician faded channel. The Nordstrom-Robinson (NR) code, a nonlinear code that has large distance for a given rate combined with a trellis code, is examined. A bound is developed on the error probability for this trellis-coded NR (TCNR) code with noncoherent reception over a frequencynonselective Rayleigh or Rician fading channel with additive white Gaussian noise (AWGN). This bound is tighter than the standard union bound. Our results indicate that the standard union bound can be significantly different from the more accurate results obtained from the improved union bound. In addition, there is a considerable coding gain at high signal-to-noise ratio (SNR) for the TCNR code over the conventional DS-SS code at the same date rate.
\end{abstract}

Index Terms - Direct-sequence spread-spectrum communications, fading channels, trellis-coded modulation.

\section{INTRODUCTION}

$\mathbf{I}$ N A CONVENTIONAL direct-sequence spread-spectrum (DS-SS) communication system, a single data bit is transmitted using a pseudorandom sequence or its negative and binary phase-shift keying (BPSK). Typically, there are $N$ chips of the pseudorandom sequence for each information bit transmitted. For a conventional system, the number of chips per information bit is a measure of the interference rejection capability of the system when used in an environment with multiple-access interference or multipath fading. The demodulation consists of (assuming perfect synchronization) a correlator matched to the pseudorandom sequence followed by a decision device. An error-correcting code such as convolutional code or block code can be used to provide additional protection, usually at the expense of data rate or processing gain. Nevertheless, better performance is usually achieved with coding.

An alternative view of this system, which makes generalizations and modifications easier to visualize, is as a stream of information bits that are encoded by a repetition code of length $N$. Each bit is repeated $N$ times. The output of the encoder is then scrambled by a pseudorandom sequence before being

Manuscript received December 1, 1995; revised March 25, 1997. This work was supported by the National Science Foundation under Grant NCR-9115969 and by the Army Research Office under Contract DAAH04-95-I-0246.

The authors are with the Department of Electrical Engineering and Computer Science Department, University of Michigan, Ann Arbor, MI 48109 USA.

Publisher Item Identifier S 0018-9545(98)03296-4. modulated using BPSK. The receiver consists of a demodulator (for each chip or code symbol) followed by a decoder, which is a soft-decision decoder for the repetition code. If an errorcorrecting code is used in addition, then essentially the system employs concatenated coding.

Viewing a DS-SS system in this manner makes it clear that one can use other codes besides the repetition code, which while having good distance properties has a poor rate. One choice is orthogonal codes. Orthogonal codes have half the distance of repetition codes, but have $N$ times as many signals. Biorthogonal codes have the same minimum distance as orthogonal codes, but can transmit one more bit of information. Biorthogonal codes, however, cannot be employed in a noncoherent system.

An $M$-ary orthogonal code of length $N, M \leq N$, has minimum distance $N / 2$. If the output of the encoder for each $\log _{2}(M)$ information bits is modulated using BPSK, the data rate is $\log _{2}(M) / N$. Thus, in general, orthogonal codes have low rate while having good distance properties. It is also important to consider the number of nearest neighbor codewords, which affect error probability, especially at low signal-to-noise ratio (SNR). For orthogonal codes, there are $\log _{2}(M)-1$ nearest neighbors. Clearly, one can use a subcode of the orthogonal code in order to reduce the number of nearest neighbors, but with no distance gain. This, however, sacrifices code rate. A method to reduce the number of nearest neighbors without sacrificing data rate is to use a combination of an orthogonal code with a trellis [1] at the expense of complexity.

In this paper, we wish to explore a coding scheme to achieve higher data rate and lower error probability. In Section II, the Nordstrom-Robinson (NR) code [3] is introduced. This nonlinear code, a member of the class of Kerdock codes [3], has good distance and rate parameters and can be efficiently decoded with a noncoherent soft-decision algorithm. The NR code has 256 codewords of length 16 with the minimum distance 6 . The 256 codewords are divided into 8 cosets, each with 32 biorthogonal codewords. For noncoherent demodulation, we consider a subcode of the NR code obtained by using only 16 orthogonal codewords in each coset. This modified NR (MNR) code has 128 codewords (or modulation vectors) of length 16 . The trellis-coded NR (TCNR) code is the MNR code combined with a four-state trellis. In Section III, we describe the TCNR code transmitted over a frequency-nonselective Rayleigh or Rician fading channel with additive white Gaussian noise. Codewords are assumed to be interleaved at every 16 chips. 
That is, every path connecting two consecutive states in the trellis corresponds to a signal from the modified NR code being transmitted over an independently faded channel. The channel is assumed to be constant over the duration of transmission of a single vector from the modified NR code. At the receiver, noncoherent reception is employed. In Section IV, we examine the error performance of the TCNR code. An upper and lower bound on the error probability are derived. In Section V, we generalize the code to also consider trellis-coded Kerdock codes. The error performance of the TCNR code and Kerdock code is compared to that of a conventional DS-SS code with the same data rate in Section VI. Finally, we present some numerical results for a pure Rayleigh fading channel, an additive white Gaussian noise channel, and a Rician fading channel in Section VII.

\section{II. $\mathrm{CODE}$}

Consider a standard, but not necessarily linear binary errorcorrecting code. The code has length $N$ and number of codewords $M$. That is, the code consists of $M$ vectors $\underline{c}_{1}, \cdots, \underline{c}_{M}$ of length $N$ from the alphabet 0,1 (in this paper $M=2^{k}$ for some integer $k$ ). The weight enumerator for the code, denoted by $A_{i}, i=0,1, \cdots, N$, is the number of codewords of (Hamming) weight $i$. For linear codes, the weight enumerator is identical to the distance distribution. This is also true for the codes considered in this paper although the code will not be linear. This property is called geometric uniformity or distance uniformity. When a code has this property, the analysis and simulation are greatly simplified because the conditional error probability does not depend on which codeword is transmitted. Thus, we can assume any particular codeword is transmitted to get the unconditional error probability. Finally, when transmitting a binary vector $\underline{c}_{i}=\left(c_{i 1}, \cdots, c_{i N}\right)$ with components 0,1 , the translation to the components in the set $+1,-1$ via $v_{i j}=(-1)^{c_{i j}}$ will take into account the usual BPSK modulation.

One class of interesting codes is the orthogonal code. This code has $M=N$ and can be formed by finding any set of orthogonal basis vectors for the space of binary $N$ vectors. One convenient set of orthogonal vectors is the Hadamard set (the orthogonal vectors are the rows of the $N$ by $N$ Hadamard matrix). Thus, the distance distribution is

$$
A_{i}= \begin{cases}1, & \text { if } i=0 \\ N-1, & \text { if } i=N / 2 .\end{cases}
$$

This code transmits $\log _{2}(N)$ bits of information using the channel $N$ times, so the rate is $\log _{2}(N) / N$.

An biorthogonal code of length 16 obtained from the Hadamard matrix plus its negative has 32 codewords of length 16 with minimum distance 8 . If we start with this biorthogonal code, we can increase the number of codewords up to 256 by carefully adding selected cosets of the biorthogonal code to the original code, with the minimum distance slightly decreasing to 6 . By doing so we get the nonlinear NR code [3]. The 256 codewords are divided into 8 cosets, each of 32 biorthogonal codewords. The geometric uniformity property is satisfied with the NR code, although it is not linear. Since the demodulator under consideration is noncoherent, only the orthogonal subcode of the NR code can be used. This subcode has 128 codewords of length 16 . The 128 codewords are divided into 8 cosets, $B_{0}, B_{1}, B_{2}, B_{3}, B_{4}, B_{5}, B_{6}$, and $B_{7}$, each of 16 orthogonal codewords. $B_{0}$ is composed of the 16row vectors in the Hadamard matrix. $B_{i}(i=1, \cdots, 7)$ is composed of the 16 codewords of weight 6 in each of the cosets of the NR code. ${ }^{1}$ This subcode is called the modified NR (MNR) code. The weight enumerator $A(z)$ of the MNR code, and the weight enumerator $A_{i}(z)$ of the coset $B_{i}(i=$ $0, \cdots, 7)$, is

$$
\begin{aligned}
& A_{0}(z)=1+15 z^{8} \\
& A_{i}(z)=16 z^{6}, \quad(i=1,2, \cdots, 7) \\
& A(z)=\sum_{i=0}^{7} A_{i}(z)=1+112 z^{6}+15 z^{8} .
\end{aligned}
$$

The distance between the codewords implies a correlation between codewords. For a certain codeword $\underline{v}_{n}$, the possible correlations are

$$
\begin{aligned}
& \left\langle\underline{v}_{n}, \underline{v}_{n}\right\rangle=16, \quad \forall n \\
& \left\langle\underline{v}_{m}, \underline{v}_{n}\right\rangle=0, \quad \underline{v}_{m}, \underline{v}_{m} \in B_{i}, m \neq n \\
& \left\langle\underline{v}_{m}, \underline{v}_{n}\right\rangle= \pm 4, \quad \underline{v}_{m} \in B_{i}, \underline{v}_{n} \in B_{j}, i \neq j
\end{aligned}
$$

where $\langle\underline{x}, \underline{y}\rangle=\sum_{i=1}^{n} x_{i} y_{i}^{*}$ denotes the correlation between vectors $\underline{x}=\left(x_{1}, \cdots, x_{n}\right)$ and $\underline{y}=\left(y_{1}, \cdots, y_{n}\right)$. If only $B_{0}$ is used, the data rate is $4 / 16$ (four information bits over 16 channel chips). By adding $B_{1}, B_{2}, \cdots, B_{7}$ to $B_{0}$, we increase the number of codewords from 16 to 128 while decreasing the minimum distance from 8 to 6 . Thus, we have decreased the minimum distance by $25 \%$ while having increased the rate by $75 \%$ to $7 / 16$. However, the number of nearest neighbors jumps to 112 , which affects the error probability. In addition, the complexity of the decoding of the NR code is roughly eight times that of the original code $B_{0}$.

If additional complexity is allowed, we can further modify the NR code to increase the minimum distance to eight and only slightly decrease the rate to 6/16. To do this, a fourstate trellis with branches labeled by the MNR codewords is used [1]. As is shown in Fig. 1, we label each of the outgoing branches of the trellis with the cosets of the MNR code to form a TCNR code, an example of finite-state codes [2]. To encode, we divided each six information bits into two and four bits. The two bits determine which coset and, hence, which outgoing branch of the trellis, while the four bits determine which codeword in that coset is transmitted. If we label the branches $B_{0}, \cdots, B_{7}$ appropriately [4], we can change the distance structure of the code to get rate $6 / 16$. The minimum distance of this new code is back to eight and the number of nearest neighbors is reduced back to 15 . Note that we have simultaneously increased the minimum distance of the code and decreased the number of nearest

\footnotetext{
${ }^{1}$ A codeword of weight six can be substituted by its negative, which is a codeword of weight ten in the same coset, with an equivalent effect in noncoherent reception. But to make the system easier to understand, we choose the codewords of weight six. This may not be practical since in real implementation we would like $B_{i}$ to be a translate of $B_{0}$, for all $i$. However, it has no effect on the performance.
} 


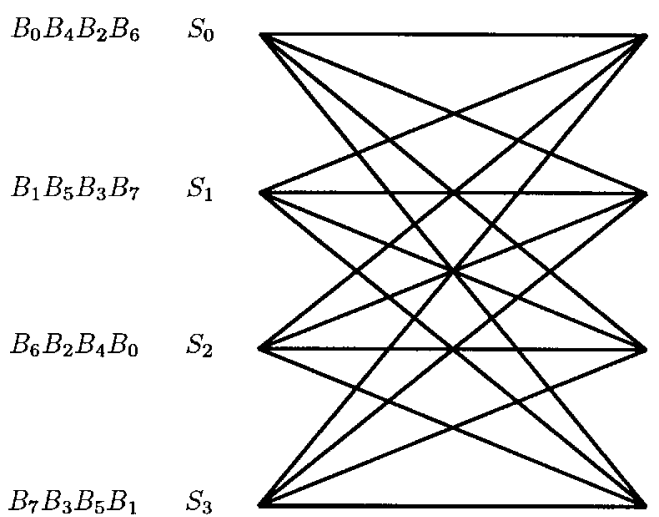

Fig. 1. Four-state trellis.

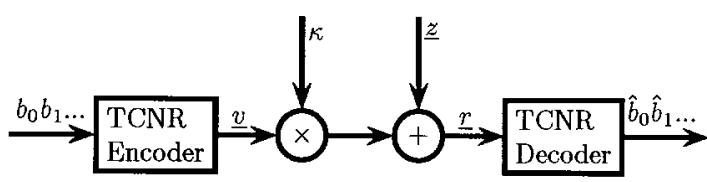

Fig. 2. Communication link.

neighbors. Furthermore, the TCNR code still has the geometric uniformity property, which makes it easy to analyze or to simulate. The disadvantage is the increase of the complexity in encoding/decoding.

\section{COMMUNiCATION System}

In this section, we describe the models used for the transmitter, channel, and receiver, as shown in Fig. 2. The data sequence to be transmitted is denoted $\left\{b_{i}\right\}$ and is assumed to be an infinite data sequence with independent identically distributed values. That is, $\operatorname{Pr}\left\{b_{i}=0\right\}=\operatorname{Pr}\left\{b_{i}=1\right\}=1 / 2$. The data sequence waveform $b(t)$ is given by

$$
b(t)=\sum_{i=-\infty}^{\infty} b_{i} p_{T_{b}}\left(t-i T_{b}\right)
$$

where $p_{T_{b}}(t)$ is the waveform $p_{T_{b}}(t)=1$ for $t \in\left[0, T_{b}\right)$ and zero elsewhere. The bit duration is $T_{b}$ and the data rate is $1 / T_{b}$. The data sequence $\left\{b_{i}\right\}$ is encoded into the coded sequence $\left\{v_{k}\right\}$ using an TCNR code. Each component $v_{k}$ of the encoder output can take values of \pm 1 .

For the TCNR code, each six bits of information plus the state of the encoder determine a sequence of length 16 to be transmitted. The duration of each component of that sequence is denoted by $T_{c}$. The waveform out of the encoder is denoted by $v(t)$ and is given by

$$
v(t)=\sum_{k=-\infty}^{\infty} v_{k} p_{T_{c}}\left(t-k T_{c}\right)
$$

where $p_{T_{\mathrm{c}}}(t)=1$ for $t \in\left[0, T_{c}\right)$ and zero elsewhere. Each 16-b codeword from the encoder is represented by a vector of length 16

$$
\underline{v}=\left(v_{1}, \cdots, v_{16}\right) \text {. }
$$

The transmitted signal is then

$$
s(t)=\sqrt{E_{s}} v(t) \cos \left(2 \pi f_{c} t+\phi\right)
$$

where $E_{s}$ is the transmitted power, $f_{c}$ is the carrier frequency (assumed to be much larger than the chip rate $1 / T_{c}$ ), and $\phi$ is a random phase unknown to the receiver, which is assumed to be fixed during each codeword duration $16 T_{c}$.

The channel is modeled as a nonselective (Rician) fading channel. In this model, the received signal $r(t)$ consists of a direct path (unfaded) signal, a diffuse (faded) signal, and additive Gaussian noise

$$
\begin{aligned}
r(t)= & c \sqrt{E_{s}} v(t) \cos \left(2 \pi f_{c} t+\phi+\theta_{c}\right) \\
& +\Gamma \sqrt{E_{s}} v(t) \cos \left(2 \pi f_{c} t+\phi+\theta_{\Gamma}\right)+n(t)
\end{aligned}
$$

where $c$ is a fixed number, $\Gamma$ is a Rayleigh random variable, and $\theta_{c}$ and $\theta_{\Gamma}$ are uniformly distributed over $[0,2 \pi)$.

The receiver processes the received signal with the usual noncoherent receiver. The output of the receiver at time $(l+1) T_{c}$ is

$$
\begin{aligned}
r_{l}= & \frac{2}{T_{c}} \int_{l T_{c}}^{(l+1) T_{c}} r(t) \cos \left(2 \pi f_{c} t\right) d t \\
& -j \frac{2}{T_{c}} \int_{l T_{c}}^{(l+1) T_{c}} r(t) \sin \left(2 \pi f_{c} t\right) d t \\
= & {\left[c e^{j\left(\phi+\theta_{c}\right)}+\Gamma e^{j\left(\phi+\theta_{\Gamma}\right)}\right] \sqrt{E_{s}} v_{l}+z_{l} }
\end{aligned}
$$

where $z_{l}$ is a zero-mean complex Gaussian random variable with variance $N_{0}$.

Without loss of generality, we employ vector notation to represent the transmitted and relevant portions of the received waveforms and the additive noise [8]. All vectors here are of length 16.

The output codeword of the TCNR encoder is $\underline{v}=$ $\left(v_{1}, \cdots, v_{16}\right)$. Notice that now $\underline{v}$ is scaled to unit length $\|\underline{v}\|^{2}=\sum_{i=1}^{16} v_{i}^{2}=1$ [8]. Therefore, $v_{k}= \pm 1 / 4, \forall k$. The transmitted signal over the channel is $\underline{s}=\sqrt{E_{s}} \underline{v}$. The energy in each codeword is $E_{s}$. Since six information bits are transmitted with each signal, $E_{s}=6 E_{b}$. We assume a nonselective fading channel with propagation gain $\kappa$

$$
\kappa=\left(c e^{j \theta}+\alpha\right)
$$

where $c$ is the unfaded component of the channel, a fixed real number, $\theta=\left(\phi+\theta_{c}\right)$ is the phase of the unfaded component, uniformly distributed over $[0,2 \pi)$, and $\alpha=\Gamma e^{\left(\phi+\theta_{\Gamma}\right)}$ is the scatter component, a complex Gaussian random variable with variance $2 \sigma^{2}$. Assume $\theta$ and $\alpha$ are independent and the channel is slow fading so $\theta$ and $\alpha$ remain constant during each symbol interval $16 T_{c}$. Thus, given $\theta, \kappa=\left(c e^{j \theta}+\alpha\right)$ is a complex Gaussian random variable with mean $c e^{j \theta}$ and variance $2 \sigma^{2}$.

The parameters $c$ and $\sigma$ may be given physical interpretations. The quantity $c$ is the strength of the direct component and $2 \sigma^{2}$ is the mean-square value of the diffuse (faded) component of the received signal. We define $\gamma^{2}=c^{2} / 2 \sigma^{2}$ as the ratio of the energy received via the direct path to the average energy of the random component. For $\gamma^{2}=\infty$, the model is that of a pure additive white Gaussian noise (AWGN) channel, while for $\gamma^{2}=0$ the channel is a pure Rayleigh faded 
channel. For situations in between $0<\gamma^{2}<\infty$, the model is that of a Rician fading channel. The average received power is related to the transmitted power via the factor $\left(c^{2}+2 \sigma^{2}\right)$. Thus, if $E_{s}$ is the energy transmitted per signal then the average received energy is

$$
\begin{aligned}
\bar{E}_{s} & =\left(c^{2}+2 \sigma^{2}\right) E_{s} \\
& =\left(\gamma^{2}+1\right) 2 \sigma^{2} E_{s} .
\end{aligned}
$$

The received signal is denoted by $\underline{r}$

$$
\underline{r}=\kappa \underline{s}+\underline{z}=\left(c e^{j \theta}+\alpha\right) \underline{s}+\underline{z}
$$

where $\kappa=\left(c e^{j \theta}+\alpha\right)$ is the propagation gain due to the channel fading and $z$ represents complex white Gaussian noise, independent of $\theta$ and $\alpha$

$$
\underline{z}=\left(z_{1}, \cdots, z_{16}\right) \text {. }
$$

The mean and variance of $z_{i}$ are given by $E\left[z_{i}\right]=0$ and $E\left[\left|z_{i}\right|^{2}\right]=N_{0}$ for all $i$.

\section{ERROR PERFORMANCE}

In this section, a lower bound and several upper bounds on the bit error probability of the TCNR code are derived. The upper bounds include the standard union bound, the union Bhattacharyya bound, and an improved union bound.

\section{A. Receiver Output}

The receiver correlates $\underline{r}$ with $\underline{v}_{i, j}$, all $i, j$, where $\underline{v}_{i, j}$ is the $j$ th codeword in the $i$ th coset $B_{i}$. Let $R_{i, j}$ represent the correlation output at the receiver, i.e., $R_{i, j}=\left\langle\underline{r}, \underline{v}_{i, j}\right\rangle$. Since the geometric uniformity property holds for the TCNR code, without loss of generality, we assume the all-zero codeword $\underline{v}_{0,0}=\left(\frac{1}{4}, \frac{1}{4}, \cdots, \frac{1}{4}\right)$, the first codeword in $B_{0}$, is transmitted. Then

$$
\begin{aligned}
& \underline{s}=\sqrt{E_{s}} \underline{v}_{0,0}=\sqrt{E_{s}}\left(\frac{1}{4}, \frac{1}{4}, \cdots, \frac{1}{4}\right) \\
& \underline{r}=\kappa \underline{s}+\underline{z}=\left(\frac{\kappa \sqrt{E_{s}}}{4}+z_{1}, \cdots, \frac{\kappa \sqrt{E_{s}}}{4}+z_{16}\right) \\
& \kappa=\left(c e^{j \theta}+\alpha\right) .
\end{aligned}
$$

The correlations of $\underline{r}$ with codewords in $B_{0}$ is as follows:

$$
\begin{aligned}
& R_{0,0}=\left\langle\underline{r}, \underline{v}_{0,0}\right\rangle=\left(c e^{j \theta}+\alpha\right) \sqrt{E_{s}}+Z_{0,0} \\
& R_{0, j}=\left\langle\underline{r}, \underline{v}_{0, j}\right\rangle=Z_{0, j}, \quad j=1,2, \cdots, 15 .
\end{aligned}
$$

The correlations of $\underline{r}$ with codewords in $B_{1}, B_{2}, \cdots$, and $B_{7}$ is

$$
\begin{gathered}
R_{i, j}=\left\langle\underline{r}, \underline{v}_{i, j}\right\rangle=\frac{\left(c e^{j \theta}+\alpha\right) \sqrt{E_{s}}}{4}+Z_{i, j}, \\
i=1, \cdots, 7, \forall j
\end{gathered}
$$

where $Z_{i, j}=\left\langle\underline{z}, \underline{v}_{i, j}\right\rangle=\sum_{n=1}^{16} \pm\left(z_{n} / 4\right)$ is a complex Gaussian random variable with $E\left[Z_{i, j}\right]=0$ and $E\left[\left|Z_{i, j}\right|^{2}\right]=$ $N_{0}, \forall i, j$. Notice that $E\left[Z_{i, j} Z_{i^{\prime}, j^{\prime}}^{*}\right]=\left\langle\underline{v}_{i, j}, \underline{v}_{i^{\prime}, j^{\prime}}\right\rangle N_{0}=$ $0, N_{0}$, or $\pm N_{0} / 4$ from (5)-(7). Thus, $R_{i, j}$ is also a complex Gaussian random variable with known mean and variance for $\forall i, j$.

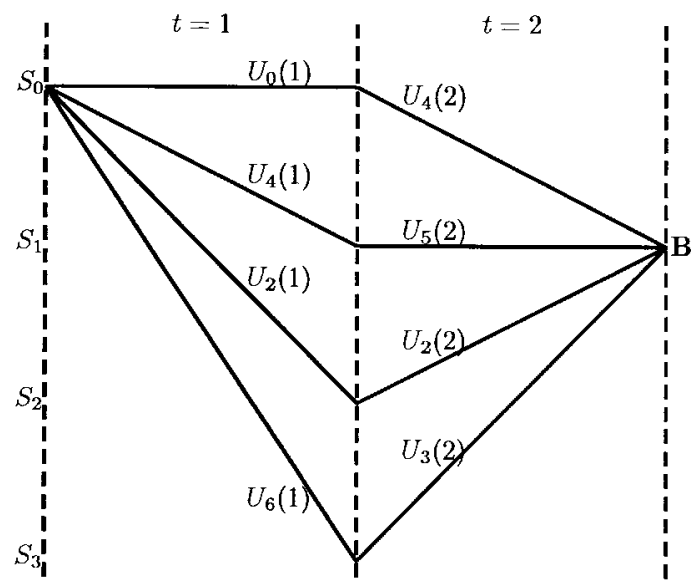

Fig. 3. Decoding at node $\mathbf{B}$.

Since noncoherent reception is assumed, the metric $U_{i}$ from a specific branch denoted by the coset $B_{i}$ is the maximum $\left\|R_{i, j}\right\|^{2}$ within that $B_{i}$

$$
\begin{aligned}
U_{i} & =\max \left(\left\|R_{i, 0}\right\|^{2},\left\|R_{i, 1}\right\|^{2}, \cdots,\left\|R_{i, 15}\right\|^{2}\right) \\
& =\max _{j}\left(\left\|\left\langle\underline{r}, \underline{v}_{i, j}\right\rangle\right\|^{2}\right) .
\end{aligned}
$$

The Viterbi decoding rule is employed. At each node of the trellis, the survivor path is the one among all the paths joining that node with maximum metric. Let $U_{i}(t)=$ the metric from $B_{i}$ at time interval $t$. For example, as is shown in Fig. 3, at node $\mathbf{B}$, the survivor path is the one with maximum among $\left[U_{0}(1)+U_{4}(2)\right],\left[U_{4}(1)+U_{5}(2)\right]$, $\left[U_{2}(1)+U_{2}(2)\right]$, and $\left[U_{6}(1)+U_{3}(2)\right]$.

As mentioned in the Introduction, every modulation vector from the modified NR code is assumed to be transmitted over an independently faded channel. Thus, after the modulation vectors are determined an interleaver that permutes the order of the modulation vectors is used. At the receiver, a similar deinterleaver is employed. Because of this, $\alpha(k)$ and $\alpha(l)$, $\theta(k)$ and $\theta(l)$, or $\underline{z}(k)$ and $\underline{z}(l)$ are independent $\forall k, l$, as long as $k \neq l$.

\section{B. Pairwise Error Probability}

First, we examine an incorrect path of length $L$ branches labeled by $L$ codewords $\underline{\mathbf{c}}_{L}=[\underline{v}(1), \underline{v}(2), \cdots, \underline{v}(L)]$. Let $\underline{\mathbf{c}}_{0}=\left[\underline{v}_{0,0}, \underline{v}_{0,0}, \cdots, \underline{v}_{0,0}\right]$, and $\underline{\mathbf{r}}_{L}=[\underline{r}(1), \underline{r}(2), \cdots, \underline{r}(L)]$ be a received vector of length $L$. The metric for the path corresponding to $\underline{\mathbf{c}}_{0}$ is

$$
W=\sum_{i=1}^{L}\left\|\left\langle\underline{r}(i), \underline{v}_{0,0}\right\rangle\right\|^{2} .
$$

The metric for the path corresponding to $\underline{\mathbf{c}}_{L}$ is

$$
V=\sum_{i=1}^{L}\|\langle\underline{r}(i), \underline{v}(i)\rangle\|^{2} .
$$

The pairwise error probability between $\underline{\mathbf{c}}_{0}$ and $\underline{\mathbf{c}}_{L}$ is given by

$$
P\left\{\underline{\mathbf{c}}_{0} \rightarrow \underline{\mathbf{c}}_{L}\right\}=\operatorname{Pr}\{W<V\} .
$$


Assume this specific path $\underline{\mathbf{c}}_{L}$ passes through $\underline{v}_{0,0} l$ times (we call these time intervals $t_{k}^{\prime}, k=1, \cdots, l$ ), through codewords $\underline{v}_{0, j} \in \hat{B}_{0}=\left(B_{0} \backslash \underline{v}_{0,0}\right) m$ times (we call these time intervals $\left.t_{k}, k=1, \cdots, m\right)$, and through codewords in $\hat{B}_{u}=\left(B_{1} \cup \cdots \cup B_{7}\right) n$ times (we call these time intervals $\left.\tau_{k}, k=1, \cdots, n\right)$ in the trellis. In other words, $\underline{v}\left(t_{1}^{\prime}\right), \cdots, \underline{v}\left(t_{l}^{\prime}\right)=v_{0,0}, \underline{v}\left(t_{1}\right), \cdots, \underline{v}\left(t_{m}\right) \in \hat{B}_{0}$, and $l+m+$ $n=L$. Note that $t_{k}^{\prime}$ 's and $t_{k}$ 's are the time it goes from state $S_{0}$ to state $S_{0}$, or from state $S_{2}$ to state $S_{3}$ in the trellis. The pairwise error probability between $\underline{\mathbf{c}}_{0}$ and $\underline{\mathbf{c}}_{L}$ depends only on $l, m$, and $n$ and not the specific codewords. Thus, the pairwise error probability between $\underline{\mathbf{c}}_{0}$ and $\underline{\mathbf{c}}_{L}$ is given by

$$
P\left\{\underline{\mathbf{c}}_{0} \rightarrow \underline{\mathbf{c}}_{L}\right\}=\operatorname{Pr}\{(W-V)<0\}=p_{l, m, n} .
$$

In order to calculate $p_{l, m, n}$, we simplify the expression for $(W-V)$. Taking into account the structure of the two codewords, we can express $W$ and $V$ as

$$
\begin{aligned}
& W=\sum_{k=1}^{l}\left\|\left\langle\underline{r}\left(t_{k}^{\prime}\right), \underline{v}_{0,0}\right\rangle\right\|^{2}+\sum_{k=1}^{m}\left\|\left\langle\underline{r}\left(t_{k}\right), \underline{v}_{0,0}\right\rangle\right\|^{2} \\
& +\sum_{k=1}^{n}\left\|\left\langle\underline{r}\left(\tau_{k}\right), \underline{v}_{0,0}\right\rangle\right\|^{2} \\
& V=\sum_{k=1}^{l}\left\|\left\langle\underline{r}\left(t_{k}^{\prime}\right), \underline{v}_{0,0}\right\rangle\right\|^{2}+\sum_{k=1}^{m}\left\|\left\langle\underline{r}\left(t_{k}\right), \underline{v}_{0, j}\right\rangle\right\|^{2} \\
& +\sum_{k=1}^{n}\left\|\left\langle\underline{r}\left(\tau_{k}\right), \underline{v}\left(\tau_{k}\right)\right\rangle\right\|^{2} .
\end{aligned}
$$

Now, by cancelling common terms, $(W-V)$ can be written as

$$
\begin{aligned}
W-V= & \sum_{k=1}^{m}\left[\left\|\left\langle\underline{r}\left(t_{k}\right), \underline{v}_{0,0}\right\rangle\right\|^{2}-\left\|\left\langle\underline{r}\left(t_{k}\right), \underline{v}_{0, j}\right\rangle\right\|^{2}\right] \\
& +\sum_{k=1}^{n}\left[\left\|\left\langle\underline{r}\left(\tau_{k}\right), \underline{v}_{0,0}\right\rangle\right\|^{2}-\left\|\left\langle\underline{r}\left(\tau_{k}\right), \underline{v}\left(\tau_{k}\right)\right\rangle\right\|^{2}\right) .
\end{aligned}
$$

Notice that $p_{l, m, n}$ is independent of $l$. Since all $\underline{r}(i)$ 's are assumed independent because of channel interleaving, $p_{l, m, n}$ can be regarded as the error probability of binary, noncoherent, square-law-combining receiver with $L$ independent, but not necessarily identical diversity. Thus, from [8] (we let $\omega=0.5$ in $[8$, eqs. (5.6a), (5.6b), and (5.6c)])

$$
p_{l, m, n} \leq \frac{1}{2} 1^{l} D_{y}^{m} D_{z}^{n}
$$

where

$$
\begin{aligned}
D_{y}= & {\left[1+\frac{1}{4}\left(\frac{2 \sigma^{2} E_{s}}{N_{0}}-1\right)\right]^{-1} } \\
& \cdot \exp \left[\frac{-\frac{1}{4} c^{2} E_{s} / N_{0}}{1+\frac{1}{4}\left(2 \sigma^{2} E_{s} / N_{0}-1\right)}\right] \\
D_{z}= & {\left[1+\frac{15}{64}\left(\frac{2 \sigma^{2} E_{s}}{N_{0}}-1\right)\right]^{-1} } \\
& \cdot \exp \left[\frac{-\frac{15}{64} c^{2} E_{s} / N_{0}}{1+\frac{15}{64}\left(2 \sigma^{2} E_{s} / N_{0}-1\right)}\right] .
\end{aligned}
$$

We can calculate the exact $p_{l, m, n}$ when $m=0$ or $n=0$. In our trellis, the only possible condition for $n=0$ is $l=n=0$ and $L=m=1$, (from state $S_{0}$ to state $S_{0}$ ), i.e., $p_{0,1,0}$, error within $B_{0}$ only, which will be discussed later. As for $m=0$, denote $p_{n}^{\prime}=p_{0,0, n}$ (this means none of the branches goes from state $S_{2}$ to state $S_{3}$ ).

1) For $\gamma^{2}=0$, that is, pure Rayleigh fading [5]

$$
p_{n}^{\prime}=\left(\frac{\lambda}{\lambda+\beta}\right)^{n} \sum_{k=0}^{n-1}\left(\begin{array}{c}
n+k-1 \\
k
\end{array}\right)\left(\frac{\beta}{\lambda+\beta}\right)^{k}
$$

where

$$
\begin{aligned}
& \lambda=\sqrt{\left(\varphi_{w}+\varphi_{v}\right)^{2} / 2-\varphi_{w v}^{2}}-\left(\varphi_{w}-\varphi_{v}\right) / 2 \\
& \beta=\sqrt{\left(\varphi_{w}+\varphi_{v}\right)^{2} / 2-\varphi_{w v}^{2}}+\left(\varphi_{w}-\varphi_{v}\right) / 2
\end{aligned}
$$

and

$$
\begin{aligned}
\varphi_{w} & =2 \sigma^{2}\left(E_{s} / N_{0}\right)+1 \\
\varphi_{v} & =\frac{2 \sigma^{2}\left(E_{s} / N_{0}\right)}{16}+1 \\
\varphi_{w v} & =\frac{2 \sigma^{2}\left(E_{s} / N_{0}\right)}{4}+\frac{1}{4} .
\end{aligned}
$$

2) For $\gamma^{2}>0$, Rician fading [6], [7], let

$$
\begin{aligned}
\beta^{\prime} & =\frac{\beta}{\varphi_{w} \varphi_{v}-\varphi_{w v}^{2}} \\
\text { and } \lambda^{\prime} & =\frac{\lambda}{\varphi_{w} \varphi_{v}-\varphi_{w v}^{2}}
\end{aligned}
$$

then

$$
\begin{aligned}
p_{n}^{\prime}= & Q\left(\frac{f r}{\sqrt{r^{2}+1}}, \frac{g}{\sqrt{r^{2}+1}}\right) \\
& +e^{-\left[\left(f^{2} r^{2}+g^{2}\right) / 2\left(r^{2}+1\right)\right]} \sum_{m=1-n}^{n-1} F_{n m}(r)\left(\frac{g r}{f}\right)^{m} \\
& \cdot I_{m}\left(\frac{f g r}{r^{2}+1}\right)
\end{aligned}
$$

where $Q(\mu, \nu)$ is Marcum's $Q$ function

$$
Q(\mu, \nu)=\int_{\nu}^{\infty} x e^{-\left(x^{2}+\mu^{2}\right) / 2} I_{0}(\mu x)
$$

and

$$
\begin{gathered}
f=\sqrt{\frac{2 n c^{2} E_{s}\left[\varphi_{v} \beta^{\prime}-1-\varphi_{w v} \beta^{\prime} / 2+\left(\varphi_{w} \beta^{\prime}+1\right) / 16\right]}{N_{0}(\lambda+\beta)}} \\
g=\sqrt{\frac{2 n c^{2} E_{s}\left[\varphi_{v} \lambda^{\prime}+1-\varphi_{w v} \lambda^{\prime} / 2+\left(\varphi_{w} \lambda^{\prime}-1\right) / 16\right]}{N_{0}(\lambda+\beta)}} \\
r=\sqrt{\lambda / \beta} \\
F_{n m}(r)=\left\{\begin{array}{cl}
-\delta_{m 0}+\left(1+\frac{1}{r^{2}}\right)^{-n} \sum_{k=m}^{n-1} \\
\cdot\left(\begin{array}{c}
n-1+m \\
k-m
\end{array}\right)\left(1+r^{2}\right)^{-k} & m \geq 0 \\
-F_{n,-m}(1 / r) & m \leq-1 .
\end{array}\right.
\end{gathered}
$$


$I_{m}(\cdot)$ is the modified Bessel function of the first kind of order $m$, and $I_{m}(\cdot)=I_{-m}(\cdot) . \delta_{m 0}$ is the Kronecker delta: $\delta_{m 0}=1$ for $m=0, \delta_{m 0}=0$ otherwise. The above indicates that $p_{n}^{\prime}$ is independent of $\theta$ 's.

\section{Weight Enumerator}

In this section, we determine the weight enumerator. To begin, redefine $H(x, y)$ to be the weight enumerator of $B_{0}$ and $G(z)$ to be the weight enumerator of the cosets $B_{1}, \cdots, B_{7}$. Usually, the weight enumerator is of the form $H(z)=1+15 z^{8},(2)$, and $G(z)=16 z^{6},(3)$, but since the system is noncoherent, this format is no longer useful. We need to employ different notations $x, y$, and $z$ in $H$ and $G$ since their metrics are different and not additively measurable in noncoherent reception. Let $x$ specify a codeword of weight zero, $y$ specify a codeword of weight eight, and $z$ specify a codeword of weight six. Thus

$$
H(x, y)=x+15 y \text {. }
$$

The first term $x$ comes from the all-zero codeword $\underline{v}_{0,0}$, and the last term $15 y$ comes from $\hat{B}_{0}=B_{0} \backslash \underline{v}_{0,0}=$ $\left\{\underline{v}_{0,1}, \underline{v}_{0,2}, \cdots, \underline{v}_{0,15}\right\}$. All 15 elements in $\hat{B}_{0}$ have the same metric, so they are all symmetric to $\underline{v}_{0,0}$ and have the same (pairwise) error probability. Similarly

$$
G(z)=16 z
$$

where $16 z$ comes from the 16 codewords in either $B_{1}, B_{2}, \cdots$, or $B_{7}$. All the 16 elements in any coset in the above have the same metric.

Each outgoing branch of the trellis is encoded with six information bits in the following way. The first two bits determine the coset of the branch and the last four bits determine which codeword in the coset

$$
\begin{aligned}
& B_{0}, B_{1}: 00 \underline{0000} \text { (determining the codewords) } \\
& B_{2}, B_{3}: 10 \underline{0000} \text { (determining the codewords) } \\
& B_{4}, B_{5}: 010000 \text { (determining the codewords) } \\
& B_{6}, B_{7}: 11 \underline{0000} \text { (determining the codewords). }
\end{aligned}
$$

In order to evaluate the standard union bound, the number of paths beginning in state $S_{0}$, diverging from state $S_{0}$, and remerging later with $l$ components equal to $\underline{v}_{0,0}, m$ components in $\hat{B}_{0}$, and $n$ components in $\hat{B}_{u}$, is needed. Let $a_{l, m, n}$ be the number of such paths. The weight enumerator then is $a(x, y, z)=\sum_{l, m, n} a_{l, m, n} x^{l} y^{m} z^{n}$. Another contribution to the weight enumerator by the transition from $S_{0}$ to $S_{0}$, will be denoted $H(x, y)=x+15 y$ indicating one path labeled $v_{0,0}, 15$ paths in $\hat{B}_{0}$, and no paths in $\hat{B}_{u}$. Thus, the weight enumerator $A(x, y, z)$ of the TCNR code is

$$
A(x, y, z)=H(x, y)+\sum_{l, m, n} a_{l, m, n} x^{l} y^{m} z^{n} .
$$

What we are really interested is the bit error probability $P_{b}$. Thus, $H$ and $G$ should be modified as follows $\left(G_{i}\right.$ is the weight enumerator of $B_{i}$ ):

$$
\begin{aligned}
H(x, y, b) & =x+y\left(4 b+6 b^{2}+4 b^{3}+b^{4}\right) \\
G_{1}(z, b) & =z\left(1+4 b+6 b^{2}+4 b^{3}+b^{4}\right)
\end{aligned}
$$

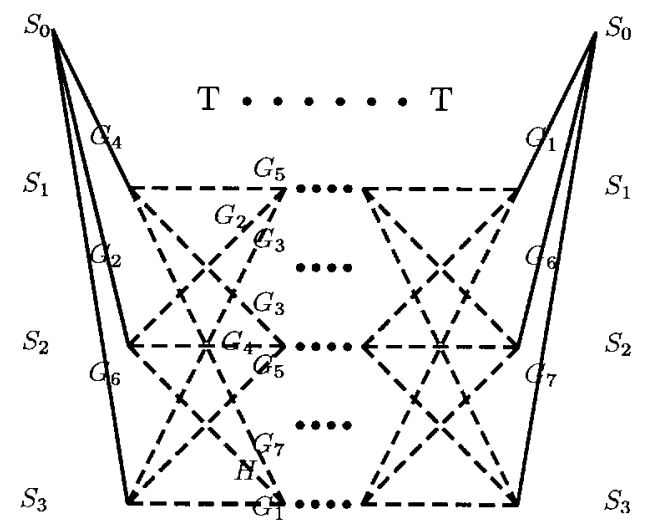

Fig. 4. Transition in the trellis.

$$
\begin{aligned}
G_{2}(z, b) & =G_{3}(z, b)=G_{4}(z, b)=G_{5}(z, b) \\
& =z\left(b+4 b^{2}+6 b^{3}+4 b^{4}+b^{5}\right)=b G_{1}(z, b) \\
G_{6}(z, b) & =G_{7}(z, b)=z\left(b^{2}+4 b^{3}+6 b^{4}+4 b^{5}+b^{6}\right) \\
& =b^{2} G_{1}(z, b) .
\end{aligned}
$$

In the above, a term $n b^{k}$ implies that there are $n$ codewords which are encoded with $k$ input ones and $(6-k)$ input zeros.

Define the overall weight enumerator $A(x, y, z, b)$ of the TCNR code, which is

$$
A(x, y, z, b)=H(x, y, b)+\sum_{l, m, n, k} a_{l, m, n, k} x^{l} y^{m} z^{n} b^{k}
$$

where the exponent of $b$ corresponds to the number of information bits $=1$ on a path and $a_{l, m, n, k}$ is the number of the $a_{l, m, n}$ paths stated above with $k$ input ones. Therefore

$$
\begin{gathered}
A(x, y, z, b)=H+\sum_{k=0}^{\infty}\left(G_{1} G_{6} G_{7}\right) T^{k}\left(G_{4} G_{2} G_{6}\right)^{t} \\
T=\left(\begin{array}{lll}
G_{5} & G_{2} & G_{3} \\
G_{3} & G_{4} & G_{5} \\
G_{7} & H & G_{1}
\end{array}\right) .
\end{gathered}
$$

The first term $H$ is from the top level path of the trellis, and the second term comes from all the divergent paths. As is shown in Fig. 4. The vector $\left(G_{4} G_{2} G_{6}\right)^{t}$ denotes the transformation from state 0 to states 1,2 , and 3 in the trellis, and the vector $\left(G_{1} G_{6} G_{7}\right)$ denotes the transformation from states 1,2 , and 3 back to state 0 . The $i$ th row in the matrix $T$ combines the transformation from states 1,2 , and 3 to state $i$. Therefore, the term $\left(G_{1} G_{6} G_{7}\right) T^{k}\left(G_{4} G_{2} G_{6}\right)^{t}$ specifies all divergent paths labeled by exactly $(k+2)$ codewords.

We know that

$$
\begin{aligned}
\sum_{k=0}^{\infty} T^{k} & =\left(I+T+T^{2}+T^{3}+\cdots\right)=(I-T)^{-1} \\
& =\left(\begin{array}{ccc}
1-G_{5} & -G_{2} & -G_{3} \\
-G_{3} & 1-G_{4} & -G_{5} \\
-G_{7} & -H & 1-G_{1}
\end{array}\right)^{-1} .
\end{aligned}
$$


Thus, after careful analysis

$$
A(x, y, z, b)=H(x, y, b)+\frac{Q_{1}(x, y, z, b)}{Q_{2}(x, y, z, b)}
$$

where

$$
\begin{aligned}
Q_{1}(x, y, z, b)= & G_{1}^{2}(z, b)\left(b^{4}+b^{3}+b\right)+G_{1}^{3}(z, b) \\
& \cdot\left(b^{6}-b^{5}-b\right)+G_{1}^{2}(z, b) H(x, y, b) b^{3} \\
Q_{2}(x, y, z, b)= & 1-G_{1}(z, b)(2 b+1)+G_{1}^{2}(z, b)\left(2 b-b^{3}\right) \\
& -G_{1}(z, b) H(x, y, b) b
\end{aligned}
$$

and $G_{1}(z, b)$ is given in (22).

\section{Standard Union Bound}

The standard union bound on the error probability is the summation of the pairwise probabilities of all the incorrect paths. From Section IV-C, we know the incorrect paths are from the following two parts.

1) Errors from $\hat{B}_{0}$ Only: For errors within $B_{0}$ only, the term $y b^{k}$ in $H(x, y, b)$ denotes an incorrect codeword in $\hat{B}_{0}$ with $k$ input ones. At any time interval $t$, let $q=p_{0,1,0}$ be the pairwise probability of $\underline{v}(t)$ to any single codeword in $\hat{B}_{0}$. This can be calculated as

$$
\begin{aligned}
q & =\operatorname{Pr}\left\{\left\|R_{0,0}(t)\right\|^{2}<\left\|R_{0,1}(t)\right\|^{2}\right\}=\cdots \\
& =\operatorname{Pr}\left\{\left\|R_{0,0}(t)\right\|^{2}<\left\|R_{0,15}(t)\right\|^{2}\right\} \\
& =\operatorname{Pr}\left\{\left\|\left[c e^{j \theta(t)}+\alpha(t)\right] \sqrt{E_{s}}+Z_{0,0}(t)\right\|^{2}<\left\|Z_{0,1}(t)\right\|^{2}\right\} \\
& =\frac{e^{-\left\{\gamma^{2} \sigma^{2}\left(E_{s} / N_{0}\right) /\left[\sigma^{2}\left(E_{s} / N_{0}\right)+1\right]\right\}}}{2 \sigma^{2}\left(E_{s} / N_{0}\right)+2} .
\end{aligned}
$$

2) Errors from All Divergent Paths: For a single divergent path labeled with $(l+m+n)$ codewords $[\underline{v}(1), \cdots, \underline{v}(l+m+$ $n)]$, where $l$ branches are labeled by $\underline{v}_{0,0}$ 's, $m$ branches are labeled by codewords in $\hat{B}_{0}$, and $n \geq 1$ branches are labeled by codewords in $\hat{B}_{u}$, its pairwise probability is bounded by $p_{l, m, n} \leq 1 / 2 D_{y}^{m} D_{z}^{n}$ (17). The corresponding term in $Q_{1}(x, y, z, b) / Q_{2}(x, y, z, b)$ of this path is $x^{l} y^{m} z^{n} b^{k}$, and the number of such paths is $a_{l, m, n, k}$.

In conclusion, the standard union bound on bit error probability $P_{b}$ is

$$
P_{b} \leq \frac{1}{6}\left[\left.\frac{\partial}{\partial b} H(x, y, b)\right|_{b=1} ^{y=q}+\sum_{l, m, n, k} k a_{l, m, n, k} p_{l, m, n}\right] .
$$

And if we take the union Bhattacharyya bound on the second component in (28)

$$
\begin{aligned}
P_{b} \leq \frac{1}{6} & {\left[\left.\frac{\partial}{\partial b} H(x, y, b)\right|_{b=1} ^{y=q}\right.} \\
& \left.+\left.\frac{\partial}{\partial b} \sum_{l, m, n, k} \frac{1}{2} a_{l, m, n, k} x^{l} y^{m} z^{n} b^{k}\right|_{x=b=1} ^{y=D_{y}, z=D_{z}}\right] \\
=\frac{1}{6} & {\left[\left.\frac{\partial}{\partial b} H(x, y, b)\right|_{b=1} ^{y=q}\right.} \\
& \left.+\frac{1}{2} \frac{\partial}{\partial b} \frac{Q_{1}(x, y, z, b)}{Q_{2}(x, y, z, b)} \mid \begin{array}{l}
y=D_{y}, z=D_{z} \\
x=b=1
\end{array}\right] .
\end{aligned}
$$

The factor $\frac{1}{6}$ comes from the fact that each codeword is encoded from six information bits.

\section{E. Improved Union Bound}

One important characteristic in the analysis of the TCNR code is that orthogonality is still preserved in $B_{0}$ even after flat fading, which is depicted in (11) and (12), $E\left[R_{0,0} R_{0, j}^{*}\right]=0$ for all $j=1,2, \cdots, 15$. Therefore, instead of pairwise error probability $q$, we can calculate the exact error probability within $\hat{B}_{0}$. Define $\operatorname{Pr}\left(\hat{B}_{0}\right)$ as

$$
\begin{aligned}
\operatorname{Pr}\left(\hat{B}_{0}\right) & =\operatorname{Pr}\left[\underline{v}(t) \in\left\{\underline{v}_{0,1}, \cdots, \underline{v}_{0,15}\right\}\right] \\
& =\operatorname{Pr}\left\{\left\|R_{0,0}(t)\right\|^{2}<\max _{j=1}^{15}\left[\left\|R_{0, j}(t)\right\|^{2}\right]\right\} .
\end{aligned}
$$

Then, from [9]

$$
\begin{aligned}
\operatorname{Pr}\left(\hat{B}_{0}\right)= & \sum_{k=1}^{15} \frac{(-1)^{k+1}\left(\begin{array}{c}
15 \\
k
\end{array}\right)}{k+1+2 k \sigma^{2}\left(\frac{E_{s}}{N_{0}}\right)} \\
& \cdot e^{\left\{-2 k \cdot \gamma^{2} \sigma^{2}\left(E_{s} / N_{0}\right) /\left[k+1+2 k \sigma^{2}\left(E_{s} / N_{0}\right)\right]\right\}} .
\end{aligned}
$$

In addition, because of symmetry

$$
\operatorname{Pr}\left[\underline{v}(t)=\underline{v}_{0,1}\right]=\cdots=\operatorname{Pr}\left[\underline{v}(t)=\underline{v}_{0,15}\right]=\frac{1}{1 \tilde{\delta}} \operatorname{Pr}\left(\hat{B}_{0}\right) .
$$

The exact bit error probability within $\hat{B}_{0}$ is thus $\frac{1}{6}\left[\frac{32}{15} \operatorname{Pr}\left(\hat{B}_{0}\right)\right]$. Therefore, following similar reasoning in Section IV-D, we obtain the improved union Bhattacharyya bound on $P_{b}$

$$
P_{b} \leq \frac{1}{6}\left[\frac{32}{15} \operatorname{Pr}\left(\hat{B}_{0}\right)+\left.\frac{1}{2} \frac{\partial}{\partial b} \frac{Q_{1}(x, y, z, b)}{Q_{2}(x, y, z, b)}\right|_{x=b=1} ^{y=D_{y}, z=D_{z}}\right] .
$$

Furthermore, let

$$
\begin{aligned}
\frac{\partial}{\partial b} \frac{Q_{1}(x, y, z, b)}{Q_{2}(x, y, z, b)}= & \sum_{k^{\prime} ; n=1}^{n=\infty} A_{0,0, n, k} z^{n} b^{k^{\prime}} \\
& +\sum_{l, m>0 ; n, k^{\prime}} A_{l, m, n, k^{\prime}} x^{l} y^{m} z^{n} b^{k^{\prime}}
\end{aligned}
$$

We can obtain an even tighter bound by calculating the exact pairwise probability $p_{n}^{\prime}$ for the first $L$ terms in $z^{n}$

$$
\begin{aligned}
& \left.\sum_{k^{\prime} ; n=1}^{n=L} A_{0,0, n, k} z^{n} b^{k^{\prime}}\right|_{z^{n}=p_{n}^{\prime}} \\
& \quad+\left.\frac{1}{2} \sum_{k^{\prime} ; n=L+1}^{n=\infty} A_{0,0, n, k} z^{n} b^{k^{\prime}}\right|_{z=D_{z}} \\
& =\sum_{k^{\prime} ; n=1}^{n=L} A_{0,0, n, k}\left(p_{n}^{\prime}-\frac{1}{2} D_{z}^{n}\right) b^{k^{\prime}} \\
& \quad+\left.\frac{1}{2} \sum_{k^{\prime} ; n=1}^{n=\infty} A_{0,0, n, k} z^{n} b^{k^{\prime}}\right|_{z=D_{z}} .
\end{aligned}
$$


Therefore, the improved union Bhattacharyya bound becomes

$$
\begin{aligned}
P_{b} \leq & \frac{16}{45} \operatorname{Pr}\left(\hat{B}_{0}\right)+\frac{1}{12} \sum_{l, m>0 ; n, k^{\prime}} A_{l, m, n, k^{\prime}} 1^{l} D_{y}^{m} D_{z}^{n} b^{k^{\prime}} \\
& +\frac{1}{6} \sum_{k^{\prime} ; n=1}^{n=L} A_{0,0, n, k} p_{n}^{\prime} b^{k^{\prime}} \\
& +\frac{1}{12} \sum_{k^{\prime} ; n=L+1}^{n=\infty} A_{0,0, n, k} D_{z}^{n} b^{k^{\prime}} \\
= & \frac{16}{45} \operatorname{Pr}\left(\hat{B}_{0}\right)+\left.\frac{1}{12} \frac{\partial}{\partial b} \frac{Q_{1}(x, y, z, b)}{Q_{2}(x, y, z, b)}\right|_{x=b=1} ^{y=D_{y}, z=D_{z}} \\
& +\frac{1}{6} \sum_{k^{\prime} ; n=1}^{n=L} A_{0,0, n, k}\left(p_{n}^{\prime}-\frac{D_{z}^{n}}{2}\right) .
\end{aligned}
$$

Notice that (32) is tighter than (31) since the last term in (32) is always nonpositive.

\section{F. Lower Bound}

Obviously, the lower bound of the error probability is from the errors in $\hat{B}_{0}$ only

$$
P_{b} \geq \frac{1}{6}\left[\frac{32}{15} \operatorname{Pr}\left(\hat{B}_{0}\right)\right]=\frac{16}{45} \operatorname{Pr}\left(\hat{B}_{0}\right) .
$$

\section{Trellis-Coded Kerdock Code of Length 64}

The code we have been examined so far, the NR code, is an example of the Kerdock codes of length 16 [3]. Now, we wish to explore our study further to the Kerdock code of length $64-\mathbf{K}_{64} \cdot \mathbf{K}_{64}$ is divided into 32 cosets, each composed of 128 biorthogonal codewords, with the minimum distance at 28 . The geometric uniformity property is still satisfied with $\mathbf{K}_{64}$. Again, only the orthogonal subcode of $\mathbf{K}_{64}$ can be used in a noncoherent system. Thus, we get a modified Kerdock code of length $64, \mathbf{K}_{64}^{\prime}$, which is divided into 32 cosets $B_{0}^{\prime}, \cdots, B_{31}^{\prime}$, and each coset contains 64 orthogonal codewords of length 64. The weight enumerator $A^{\prime}(z)$ of $\mathbf{K}_{64}^{\prime}$, and the weight enumerator $A_{i}^{\prime}(z)$ of the coset $B_{i}^{\prime}(i=0, \cdots, 31)$, is as follows:

$$
\begin{aligned}
& A_{0}^{\prime}(z)=1+63 z^{32} \\
& A_{i}^{\prime}(z)=64 z^{28}, \quad(i=1,2, \cdots, 31) \\
& A^{\prime}(z)=\sum_{i=0}^{31} A_{i}^{\prime}(z)=1+1984 z^{28}+63 z^{32} .
\end{aligned}
$$

When $\mathbf{K}_{64}^{\prime}$ is combined with a fully connected 16-state trellis shown in Fig. 5, we obtain a trellis-coded Kerdock (TCK) code of length 64 (TCK64). In the TCK64 code, 10 $\mathrm{b}$ of information is transmitted in 64 channel chips, and thus the rate of the TCK64 code is 10/64.

Following similar procedures in Section IV, we obtain the union bound, the improved union bound, and the lower bound of the TCK64 code over a Rayleigh or Rician fading channel.

\section{COMPARISON OF TCNR WITH A CONVENTIONAL DS-SS}

In this section, we look at the asymptotic performance at high SNR's and compare the performance of a TCNR code and trellis-coded Kerdock code with an orthogonal code.
$\begin{array}{llllllllllllllllllllll}B_{0}^{\prime} & B_{1}^{\prime} & B_{2}^{\prime} & B_{3}^{\prime} & B_{4}^{\prime} & B_{5}^{\prime} & B_{6}^{\prime} & B_{7}^{\prime} & B_{8}^{\prime} & B_{9}^{\prime} & B_{10}^{\prime} B_{11}^{\prime} B_{12}^{\prime} B_{13}^{\prime} B_{14}^{\prime} B_{15}^{\prime} S_{0} & B_{16}\end{array}$ $B_{16}^{\prime} B_{17}^{\prime} B_{18}^{\prime} B_{19}^{\prime} B_{20}^{\prime} B_{21}^{\prime} B_{22}^{\prime} B_{23}^{\prime} B_{24}^{\prime} B_{25}^{\prime} B_{26}^{\prime} B_{27}^{\prime} B_{28}^{\prime} B_{29}^{\prime} B_{30}^{\prime} B_{31}^{\prime} S_{1}$ $B_{12}^{\prime} B_{13}^{\prime} B_{14}^{\prime} B_{15}^{\prime} B_{0}^{\prime} B_{1}^{\prime} B_{2}^{\prime} B_{3}^{\prime} B_{4}^{\prime} B_{5}^{\prime} B_{6}^{\prime} B_{7}^{\prime} B_{8}^{\prime} B_{9}^{\prime} B_{10}^{\prime} B_{11}^{\prime} S_{2}$ $B_{28}^{\prime} B_{29}^{\prime} B_{30}^{\prime} B_{31}^{\prime} B_{16}^{\prime} B_{17}^{\prime} B_{18}^{\prime} B_{19}^{\prime} B_{20}^{\prime} B_{21}^{\prime} B_{22}^{\prime} B_{23}^{\prime} B_{24}^{\prime} B_{25}^{\prime} B_{26}^{\prime} B_{27}^{\prime}$ $\begin{array}{llllllllll}B_{8}^{\prime} & B_{9}^{\prime} & B_{10}^{\prime} B_{11}^{\prime} B_{12}^{\prime} B_{13}^{\prime} B_{14}^{\prime} B_{15}^{\prime} B_{0}^{\prime} & B_{1}^{\prime} & B_{2}^{\prime} & B_{3}^{\prime} & B_{4}^{\prime} & B_{5}^{\prime} & B_{6}^{\prime} & B_{7}^{\prime}\end{array}$ $B_{24}^{\prime} B_{25}^{\prime} B_{26}^{\prime} B_{27}^{\prime} B_{28}^{\prime} B_{29}^{\prime} B_{30}^{\prime} B_{31}^{\prime} B_{16}^{\prime} B_{17}^{\prime} B_{18}^{\prime} B_{19}^{\prime} B_{20}^{\prime} B_{21}^{\prime} B_{22}^{\prime} B_{23}^{\prime} S_{5}$ $B_{4}^{\prime} B_{5}^{\prime} B_{6}^{\prime} B_{7}^{\prime} B_{8}^{\prime} B_{9}^{\prime} B_{10}^{\prime} B_{11}^{\prime} B_{12}^{\prime} B_{13}^{\prime} B_{14}^{\prime} B_{15}^{\prime} B_{0}^{\prime} B_{1}^{\prime} B_{2}^{\prime} B_{3}^{\prime} S_{0}$ $B_{20}^{\prime} B_{21}^{\prime} B_{22}^{\prime} B_{23}^{\prime} B_{24}^{\prime} B_{25}^{\prime} B_{26}^{\prime} B_{27}^{\prime} B_{28}^{\prime} B_{29}^{\prime} B_{30}^{\prime} B_{31}^{\prime} B_{16}^{\prime} B_{17}^{\prime} B_{18}^{\prime} B_{19}^{\prime}$ $B_{15}^{\prime} B_{14}^{\prime} B_{13}^{\prime} B_{12}^{\prime} B_{11}^{\prime} B_{10}^{\prime} B_{9}^{\prime} B_{8}^{\prime} B_{7}^{\prime} B_{6}^{\prime} B_{5}^{\prime} B_{4}^{\prime} B_{3}^{\prime} B_{2}^{\prime} B_{1}^{\prime} B_{0}^{\prime} S_{8}$ $B_{31}^{\prime} B_{30}^{\prime} B_{29}^{\prime} B_{28}^{\prime} B_{27}^{\prime} B_{26}^{\prime} B_{25}^{\prime} B_{24}^{\prime} B_{23}^{\prime} B_{22}^{\prime} B_{21}^{\prime} B_{20}^{\prime} B_{19}^{\prime} B_{18}^{\prime} B_{17}^{\prime} B_{16}^{\prime} S_{9}$ $B_{11}^{\prime} B_{10}^{\prime} B_{9}^{\prime} B_{8}^{\prime} B_{7}^{\prime} B_{6}^{\prime} B_{5}^{\prime} B_{4}^{\prime} B_{3}^{\prime} B_{2}^{\prime} B_{1}^{\prime} B_{0}^{\prime} B_{15}^{\prime} B_{14}^{\prime} B_{13}^{\prime} B_{12}^{\prime} S_{10}$ $B_{27}^{\prime} B_{26}^{\prime} B_{25}^{\prime} B_{24}^{\prime} B_{23}^{\prime} B_{22}^{\prime} B_{21}^{\prime} B_{20}^{\prime} B_{19}^{\prime} B_{18}^{\prime} B_{17}^{\prime} B_{16}^{\prime} B_{31}^{\prime} B_{30}^{\prime} B_{29}^{\prime} B_{28}^{\prime} S_{1}$ $\begin{array}{lllllllllllll}B_{7}^{\prime} & B_{6}^{\prime} & B_{5}^{\prime} & B_{4}^{\prime} & B_{3}^{\prime} & B_{2}^{\prime} & B_{1}^{\prime} & B_{0}^{\prime} & B_{15}^{\prime} B_{14}^{\prime} B_{13}^{\prime} B_{12}^{\prime} B_{11}^{\prime} B_{10}^{\prime} B_{9}^{\prime} B_{8}^{\prime} & S_{12}\end{array}$ $B_{23}^{\prime} B_{22}^{\prime} B_{21}^{\prime} B_{20}^{\prime} B_{19}^{\prime} B_{18}^{\prime} B_{17}^{\prime} B_{16}^{\prime} B_{31}^{\prime} B_{30}^{\prime} B_{29}^{\prime} B_{28}^{\prime} B_{27}^{\prime} B_{26}^{\prime} F_{25}^{\prime} B_{24}^{\prime} S$ $\begin{array}{llllllllll}B_{3}^{\prime} & B_{2}^{\prime} & B_{1}^{\prime} & B_{0}^{\prime} & B_{15}^{\prime} B_{14}^{\prime} B_{13}^{\prime} B_{12}^{\prime} B_{11}^{\prime} B_{10}^{\prime} B_{9}^{\prime} & B_{8}^{\prime} & B_{7}^{\prime} & B_{6}^{\prime} & B_{5}^{\prime} & B_{4}^{\prime}\end{array}$ $B_{19}^{\prime} B_{18}^{\prime} B_{17}^{\prime} B_{16}^{\prime} B_{31}^{\prime} B_{30}^{\prime} B_{29}^{\prime} B_{28}^{\prime} B_{27}^{\prime} B_{26}^{\prime} B_{25}^{\prime} B_{24}^{\prime} B_{23}^{\prime} B_{22}^{\prime} B_{21}^{\prime} B_{20}^{\prime} S_{15}$

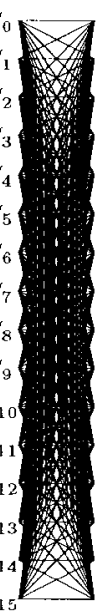

Fig. 5. 16-state trellis.

First, consider a conventional DS-SS system using an 8-ary orthogonal code $C_{8}$, where each codeword in $C_{8}$ consists of eight chips. If the chip duration of $C_{8}$ is the same as that of the TCNR code, then both codes will have the same data rate. This is because in $C_{8}, 3 \mathrm{~b}$ of information are transmitted over eight channel chips, which makes the rate of $C_{8} 3 / 8$, the same as that of the TCNR code, 6/16. Therefore, a fair comparison can be obtained between the $C_{8}$ and the TCNR code. Similarly the TCK code which has rate 10/64 can be compared to a 32ary orthogonal code with five information bits. Also, the TCK code which has rate 14/256 can be compared to the 128-ary orthogonal code of rate $7 / 128$.

Consider the comparison of 8-ary orthogonal with the TCNR code. The receiver output when using $C_{8}$ is

$$
\begin{aligned}
& R_{0}=\left(c e^{j \theta}+\alpha\right) \sqrt{E_{s}}+Z_{0} \\
& R_{j}=Z_{j}, \quad j=1,2, \cdots, 7
\end{aligned}
$$

where $E_{s}=3 E_{b}$. Thus, following the same procedure in (30), we can get the exact bit error probability $P_{b}\left(C_{8}\right)$ [9]

$$
\begin{aligned}
P_{b}\left(C_{8}\right)= & \frac{4}{7} \sum_{k=1}^{7} \frac{(-1)^{k+1}\left(\begin{array}{l}
7 \\
k
\end{array}\right)}{k+1+2 k \sigma^{2}\left(\frac{E_{s}}{N_{0}}\right)} \\
& \cdot e^{\left\{-2 k \gamma^{2} \sigma^{2}\left(E_{s} / N_{0}\right) /\left[k+1+2 k \sigma^{2}\left(E_{s} / N_{0}\right)\right]\right\}} .
\end{aligned}
$$

The factor $\frac{4}{7}$ comes from the fact that for an $M$-ary orthogonal code, $P_{b}=\left(2^{M-1} / 2^{M}-1\right) P_{\text {codeword }}$.

We can determine the performance at high SNR quite easily. For pure Rayleigh fading from (30), (33), and (39), the asymptotic approach of $P_{b}$ at high SNR of is (we normalize the energy to $E_{b}$ in the following):

$$
\begin{aligned}
P_{b}(\mathrm{TCNR}) & \simeq \frac{16}{45}\left[\sum_{k=1}^{15} \frac{(-1)^{k+1}\left(\begin{array}{c}
15 \\
k
\end{array}\right)}{k}\right]\left(\frac{1}{12 \sigma^{2}}\right)\left(\frac{E_{b}}{N_{0}}\right)^{-1} \\
& \simeq\left(\frac{0.0893}{\sigma^{2}}\right)\left(\frac{E_{b}}{N_{0}}\right)^{-1}
\end{aligned}
$$




$$
\begin{aligned}
P_{b}\left(C_{8}\right) & \simeq \frac{4}{7}\left[\sum_{k=1}^{7} \frac{(-1)^{k+1}\left(\begin{array}{l}
7 \\
k
\end{array}\right)}{k}\right]\left(\frac{1}{6 \sigma^{2}}\right)\left(\frac{E_{b}}{N_{0}}\right)^{-1} \\
& \simeq\left(\frac{0.247}{\sigma^{2}}\right)\left(\frac{E_{b}}{N_{0}}\right)^{-1} .
\end{aligned}
$$

The above indicates that there is a $4.0-\mathrm{dB}$ gain in $E_{b} / N_{0}$ at high SNR in pure Rayleigh fading. As has been expected, the TCNR code has better performance, at the expense of trellis complexity.

For white Gaussian noise and high SNR, the performance of the TCNR code is $3 \mathrm{~dB}$ better than the orthogonal code of the same rate. This is due to the fact that for a fixed energy per bit the energy per symbol in the 8-ary orthogonal case is $3 E_{b}$ while in the TCNR case is $6 E_{b}$.

For the Kerdock codes a similar comparison can be made. However, the computation using an expression involving an alternating series does not yield a stable algorithm to compute the error probability. Instead a standard integral expression is used. Let $M$ be the size of the orthogonal set of codewords. Let $k$ be the number of bits for the orthogonal scheme where $k=$ $\log _{2}(M)$. For the 16-ary orthogonal scheme, if we employ $B_{0}$ only, which is a conventional DS-SS orthogonal scheme, $k=4$ bits of information are transmitted in each codeword, with codeword probability $P_{e, O}$ and bit error probability $P_{b, O}$. For pure Rayleigh fading and high SNR the symbol error probability is given by

$$
P_{e, O} \cong \frac{k a(M)}{E_{s} / N_{0}}
$$

where

$$
a(M)=\frac{1}{\log _{2}(M)} \sum_{n=1}^{M-1}(-1)^{(n+1)} \frac{\left(\begin{array}{c}
M-1 \\
n
\end{array}\right)}{n} .
$$

The energy per bit is related to the energy per symbol by $\bar{E}_{s}=k \bar{E}_{b}$. The bit error probability $P_{b, O}$ is related to the symbol error probability by

$$
P_{b, O}=\frac{M}{2(M-1)} P_{e, O} .
$$

Thus, the bit error probability is related to the energy per bit by

$$
P_{b, O} \cong \frac{M a(M)}{2(M-1)}\left(\frac{\bar{E}_{b}}{N_{0}}\right)^{-1} .
$$

The above shows, at high SNR, $P_{b, O}$ is proportional to the inverse of SNR. The constant $a(M)$ starts out as one for $M=2$, but approaches $\ln (2)$ for $M$ large.

For comparison purposes consider a trellis code using a union of translates of an orthogonal set of size $2 M$. Each branch in the trellis is labeled by a coset of an orthogonal set of size $2 M$. The minimum distance codewords form an orthogonal code. The TCNR code or TCK code can transmit $k+1+l$ bits where $l$ bits determine which path in the trellis to take while $k+1$ bits determine which signal in the coset of an orthogonal set to transmit. For example, the trellis-coded
Kerdock code using translates of a 64-ary signal set with $l=4$ bits determining the path through the trellis and $k+1=6$ bits determining the transmitted signal along a particular path has rate $10 / 64$. The orthogonal set of size 32 has rate $5 / 32$.

The error probability of the trellis-coded system at high SNR's is dominated by error events at the minimum distance. These error events are errors within the coset of the orthogonal code. Thus, the symbol error probability for these events is

$$
P_{e, T} \cong \frac{(k+1) a(2 M)}{\bar{E}_{s} / N_{0}} .
$$

Now, $(k+1+l) \bar{E}_{b}=\bar{E}_{s}$. Thus

$$
P_{e, T} \cong \frac{(k+1) a(2 M)}{(k+1+l) \bar{E}_{b} / N_{0}} .
$$

Also, of the $k+1+l$ bits only $k+1$ can be in error for a minimum distance error event. Thus

$$
P_{b, T} \cong\left[\frac{k+1}{k+l+1}\right]^{2} \frac{2 M}{2(2 M-1)} \frac{a(2 M)}{\bar{E}_{b} / N_{0}} .
$$

For the same bit error probability the SNR needed is reduced for the trellis-coded system by the factor

$$
G=\left[\frac{k+l+1}{k+1}\right]^{2} \frac{(2 M-1)}{(2 M-2)} \frac{a(M)}{a(2 M)} .
$$

From the above, by finding the value of $G$, we may predict the performance of other Kerdock codes [1], [3] in trellis-coded version with noncoherent reception. For the case of length 64 Kerdock codes the gain is $4.60 \mathrm{~dB}$ while for the case of length 256 Kerdock codes the gain is $4.93 \mathrm{~dB}$. Of course, the gain is achieved with significantly more complex receivers.

\section{NUMERICAL RESULTS}

In the figures to follow, we plot the bounds on the bit error probability versus the average received SNR. In Fig. 6, the curves of the union bound, the improved union bound, and the lower bound are shown for the case of the TCNR code over a Rayleigh fading channel $\left(\gamma^{2}=0\right)$. We take $L=20$ in (32) to get the improved union bound. Simulation results are also shown in the figure. For the same Rayleigh fading channel, the improved union bound, the lower bound, the simulation results, and the error performance of the conventional DS-SS code with the same rate, are shown in Fig. 7. In Fig. 8, the curves of the union bound, the improved union bound, and the lower bound are shown for the case of the TCNR code over a Rician fading channel with $\gamma^{2}=7$ [we take $L=10$ in (32) to get the improved union bound]. Simulation results are also shown in the figure. For the same Rician fading channel, the improved union bound, the lower bound, the simulation results, and the error performance of the conventional DS-SS code with the same rate, are shown in Fig. 9. From Figs. 6 and 8, we can see that the improved union bound is a fairly tight upper bound at high SNR in either Rayleigh or Rician channel fading. This is because at high SNR, most terms in $A(x, y, z, b)$ except 


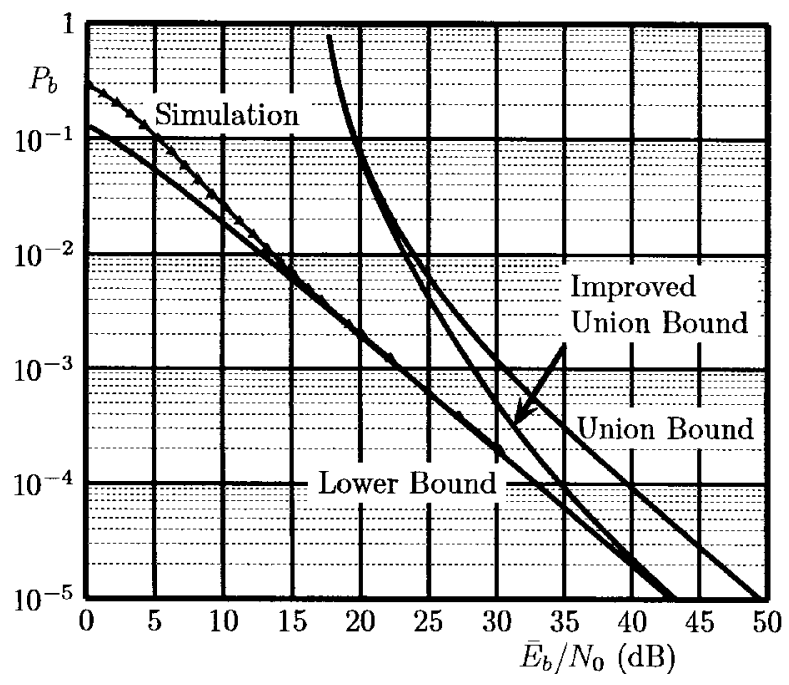

Fig. 6. Error probability of TCNR over a Rayleigh faded channel $\left(\gamma^{2}=0\right)$.

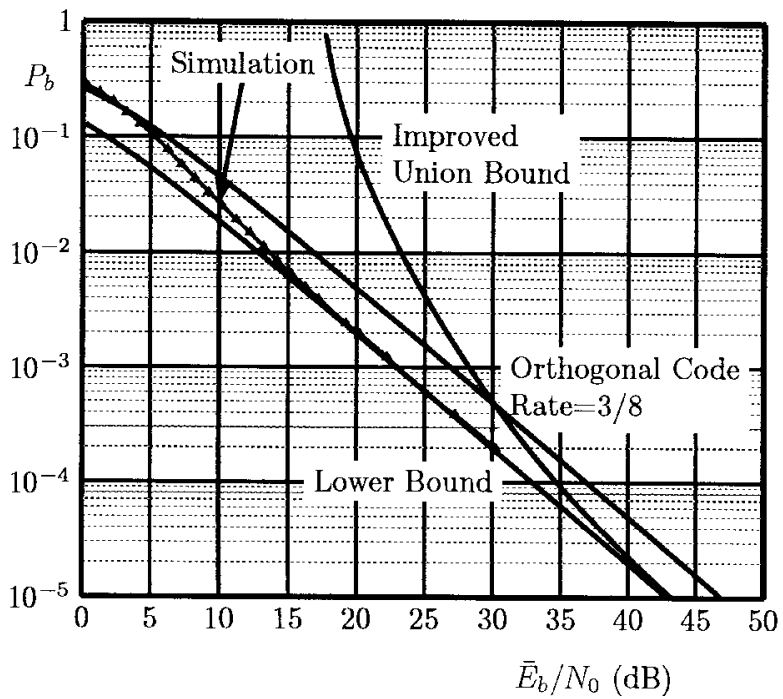

Fig. 7. Comparison of error probabilities of TCNR and orthogonal modulation over a Rayleigh faded channel $\left(\gamma^{2}=0\right)$.

$H(x, y, b)$ vanish, i.e., the errors are from $\hat{B}_{0}$ only, which is also our lower bound.

At high SNR, the asymptotic performance of the union bound is

$$
\left.\frac{1}{6} \frac{\partial}{\partial b} H(x, y, b)\right|_{x=q, b=1}=\frac{16}{3} q \simeq \frac{4}{9}\left(\frac{1}{\sigma^{2}}\right)\left(\frac{E_{b}}{N_{0}}\right)^{-1}
$$

and the asymptotic performance of the improved union bound is

$$
\begin{aligned}
\left.\frac{1}{6} \frac{\partial}{\partial b} H(x, y, b)\right|_{x=\operatorname{Pr}\left(\hat{B}_{0}\right) / 15, b=1} & =\frac{16}{45} \operatorname{Pr}\left(\hat{B}_{0}\right) \\
& \simeq\left(\frac{0.098318}{\sigma^{2}}\right)\left(\frac{E_{b}}{N_{0}}\right)^{-1} .
\end{aligned}
$$

Thus, for pure Rayleigh fading and high SNR the improved union bound is tighter by $6.56 \mathrm{~dB}$ than the standard union bound.

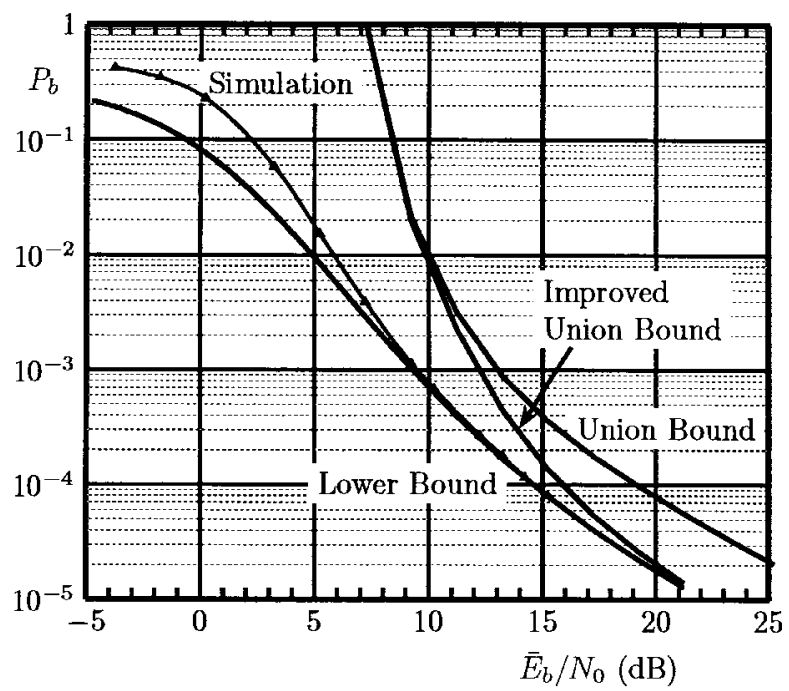

Fig. 8. Error probability of TCNR over a Rician faded channel $\left(\gamma^{2}=7\right)$.

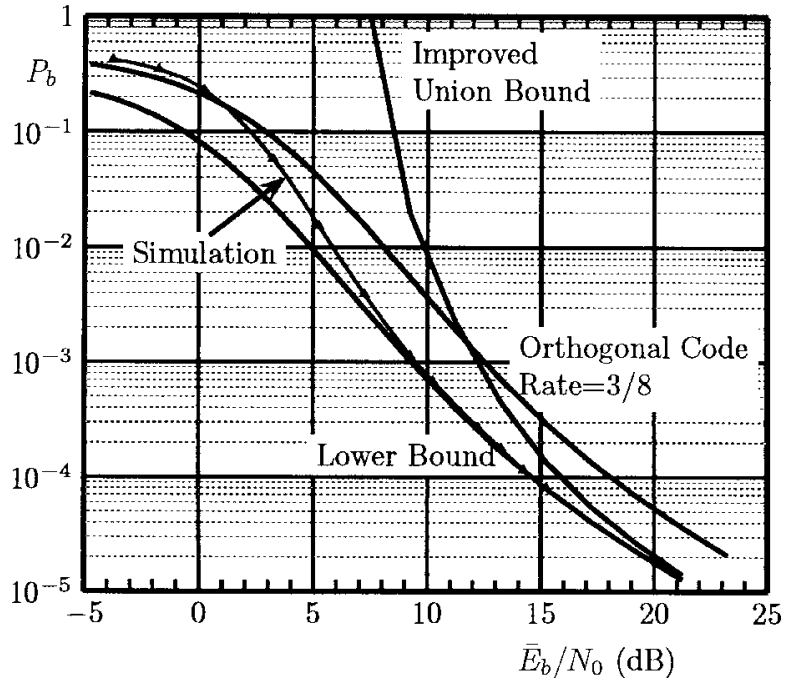

Fig. 9. Comparison of error probabilities of TCNR and orthogonal modulation over a Rician faded channel $\left(\gamma^{2}=7\right)$.

Finally, the performance of the TCNR code over an AWGN channel with no fading and its comparison with the conventional DS-SS code is shown in Figs. 10 and 11. From Fig. 10 , we can see that now the standard union bound is barely separable from the improved union bound. Fig. 11 shows that the TCNR code has a 3-dB improvement in $E_{b} / N_{0}$ over the conventional DS-SS code.

In Figs. 12 and 14, the curves of the union bound, the improved union bound, and the lower bound are shown for the case of the TCK64 code over a Rayleigh fading channel $\left(\gamma^{2}=0\right)$ and a Rician fading channel $\left(\gamma^{2}=7\right)$. The comparison of the TCK64 code and a conventional DS-SS code with 32-ary orthogonal codewords of length 32, which has rate 5/32, is shown in Figs. 13 and 15. There is a 4.6013$\mathrm{dB}$ gain in $E_{b} / N_{0}$ at high SNR. Similar to the TCNR code, the TCK64 code has better performance than the conventional DS-SS code. 


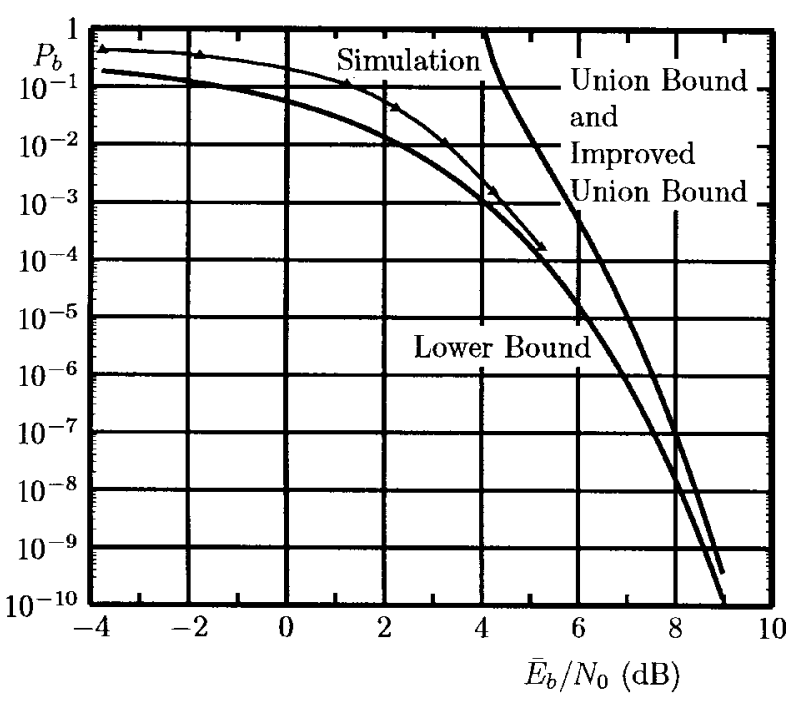

Fig. 10. Error probability of TCNR and orthogonal modulation over an AWGN channel $\left(\gamma^{2}=\infty\right)$.

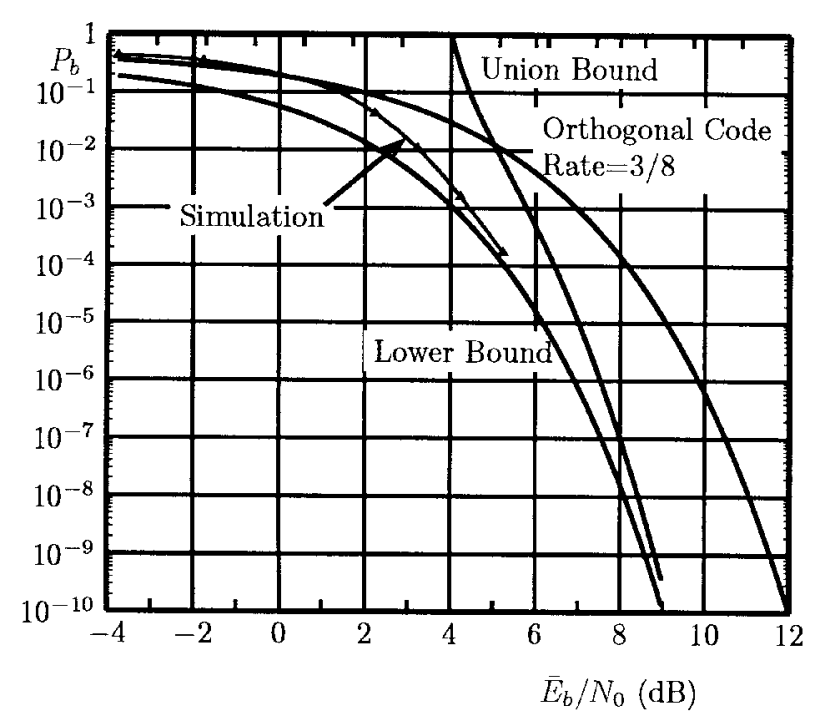

Fig. 11. Comparison of error probabilities of TCNR and orthogonal modulation over an AWGN channel $\left(\gamma^{2}=\infty\right)$.

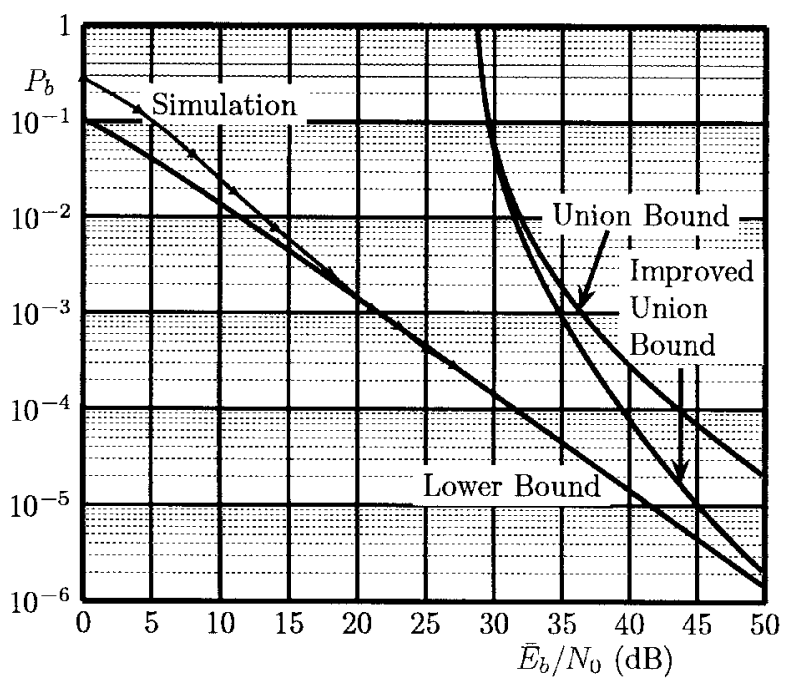

Fig. 12. Error probabilities of TCK and orthogonal modulation over Rayleigh faded channel $\left(\gamma^{2}=0\right)$.

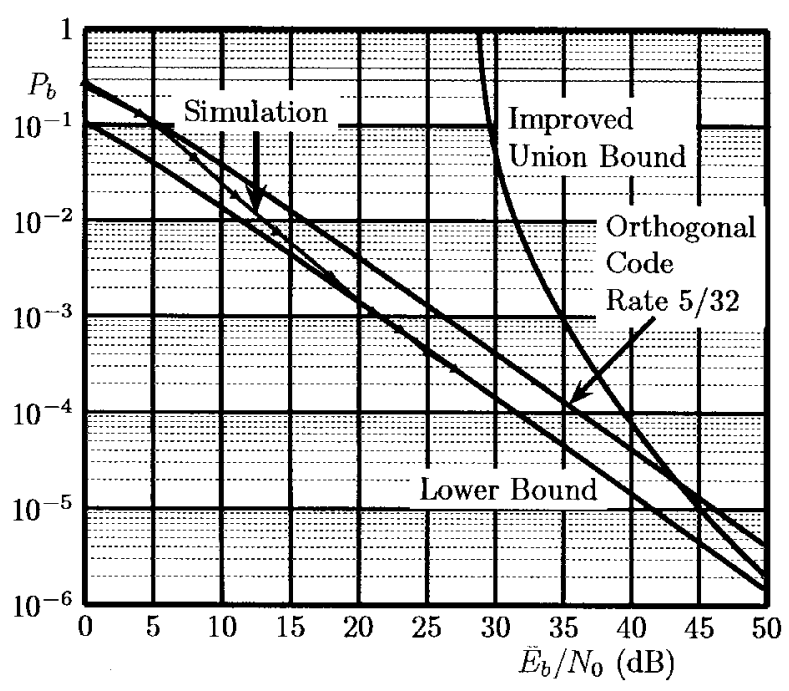

Fig. 13. Comparison of error probabilities of TCK code and orthogonal modulation over a Rayleigh faded channel $\left(\gamma^{2}=0\right)$.

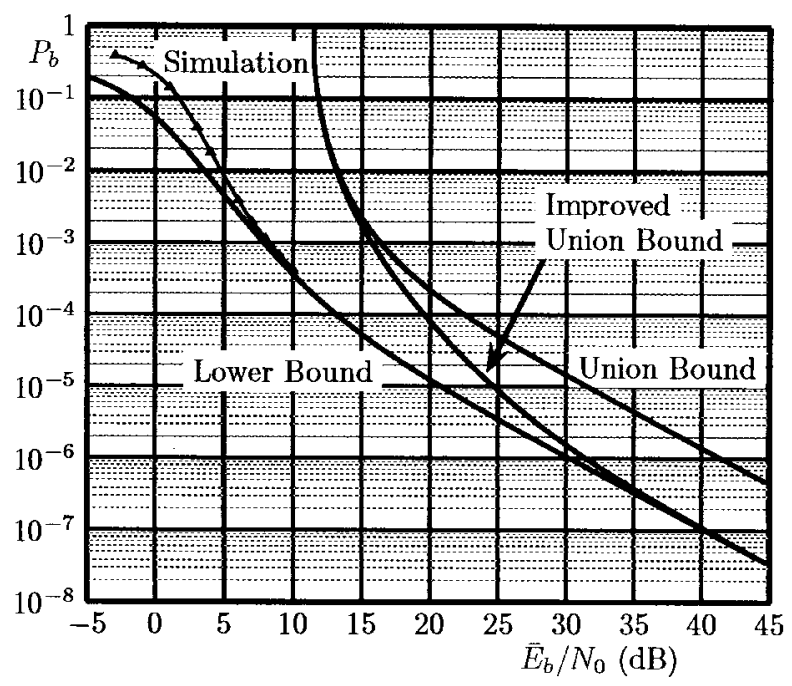

Fig. 14. Error probabilities of TCK and orthogonal modulation over a Rician faded channel $\left(\gamma^{2}=7\right)$.

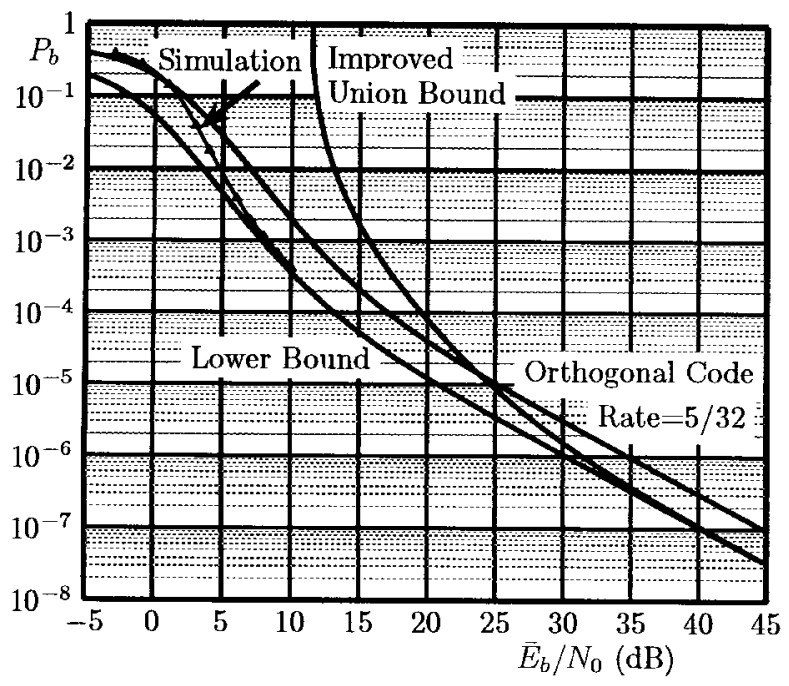

Fig. 15. Comparison of error probabilities of TCK code and orthogonal modulation over a Rician faded channel $\left(\gamma^{2}=7\right)$. 


\section{CONCLUSION}

In this work, the upper and lower bounds on the error probability have been derived for a different schemes for DS-SS, the NR code in a trellis-coded version, over a nonselective fading channel with noncoherent reception. Our results indicate that the improved union bound we have derived performs a more accurate upper bound than the standard union bound. This is because we can calculate the exact $\operatorname{Pr}\left(\hat{B}_{0}\right)$ for arbitrary $\gamma^{2}$. Our results also indicate that the TCNR code has a better error performance than a conventional DS-SS at the same data rate.

Larger gains than those shown above can be achieved by combining the MNR code with a 8-state or even up to a 64state trellis by dividing the cosets into subsets. This is believed to achieve a lower error probability while maintaining the same data rate. However, the complexity increases with the number of states. Similar possibilities for the trellis-coded Kerdock code exist as well. Another important issue that needs to be addressed is the performance in a frequency-selective fading channel, in which the orthogonality in each coset may no longer be obtained after transmission.

\section{REFERENCES}

[1] W. E. Stark and J. S. Lehnert, "Coding alternatives for direct-sequence spread-spectrum multiple access communications," in 32nd Annu. Allerton Conf. Communication, Control, and Computing, Oct. 1994, pp. 454-463.

[2] F. Pollara, R. J. McEliece, and K. Abdel-Ghaffer, "Finite state codes," IEEE Trans. Inform. Theory, vol. 34, pp. 1083-1089, Sept. 1988.

[3] F. J. MacWilliams and N. J. Sloane, The Theory of Error-Correcting Code. Amsterdam: North-Holland, 1978.

[4] J. G. Proakis, Digital Communications, 2nd ed. New York: McGrawHill, 1989.

[5] J. Omura and T. Kailath, "Some useful probability distribution," Systems Theory Lab., Stanford Univ., Stanford, CA, Tech. Rep. 7050-6, Sept. 1965.
[6] P. A. Bello, "Binary error probabilities over selectively fading channels containing specular components," IEEE Trans. Commun. Technol., vol. COM-14, no. 4, pp. 400-406, 1966.

[7] R. Price, "Some noncentral F-Distributions expressed in closed form," Biometrika, vol. 51, pp. 107-122, June 1964.

[8] I. M. Jacobs, "Probability-of-error bounds for binary transmission on the slowly fading Rician channel," IEEE Trans. Inform. Theory, vol. IT-12, pp. 431-441, Oct. 1966.

[9] W. C. Lindsey, "Error probabilities for Rician fading multichannel reception of binary and $N$-ary signals," IEEE Trans. Inform. Theory, vol. IT-10, pp. 339-350, Oct. 1964.

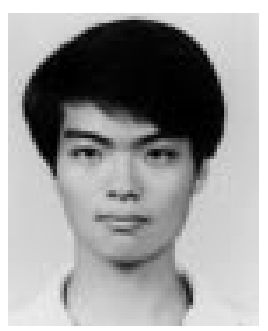

Victor Wen-Kai Cheng received the B.S. degree from National Taiwan University, Taipei, Taiwan, in 1989 and the M.S. degree from the University of Michigan, Ann Arbor, in 1995, both in electrical engineering. He is currently working toward the Ph.D. degree at the University of Michigan.

His research interests are in the areas of coding and communication theory, especially for spreadspectrum schemes over fading channels.

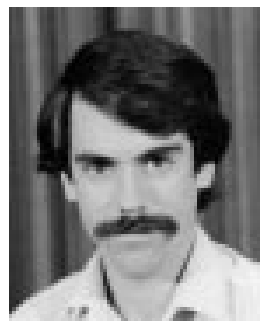

Wayne E. Stark received the B.S. (with highest honors), M.S., and Ph.D. degrees in electrical engineering from the University of Illinois, Urbana, in 1978, 1979, and 1982, respectively.

Since September 1982, he has been a Faculty Member in the Department of Electrical Engineering and Computer Science, University of Michigan, Ann Arbor. His research interests are in the areas of coding and communication theory, especially for spread-spectrum and wireless communication networks.

Dr. Stark was selected by the National Science Foundation as a 1985 Presidential Young Investigator. He is a Member of Eta Kappa Nu, Phi Kappa Phi, and Tau Beta Pi. From 1984 to 1989, he was Editor for Communication Theory of the IEEE TRANSACTIONS ON COMMUNICATIONS in the area of spreadspectrum communications. He was involved in the planning and organization of the 1986 International Symposium on Information Theory, which was held in Ann Arbor. 